\title{
DENSE PERIODICITY ON THE INTERVAL
}

\author{
MARCY BARGE AND JOE MARTIN
}

ABSTRACT. We give a description of those continuous functions on the interval for which the set of periodic points is dense.

The purpose of this paper is to describe those continuous functions $f$ on the interval for which the set of periodic points is dense. While this description could have been given in terms of $f$, it is simpler and more informative to describe $f^{2}$.

Suppose that $I$ is an interval, $f: I \rightarrow I$ is continuous, and that the set of periodic points of $f$ is dense in $I$. What follows is a rough description of the graph of $f^{2}$ : on the diagonal there are "blocks of orbit density," intervals $J_{i}$ such that $f^{2}\left(J_{i}\right)=J_{i}$. For each $i$, the restriction of $f^{2}$ to $J_{i}$ has a point whose orbit is dense in $J_{i}$. Furthermore, if $x \in I-\bigcup_{1}^{\infty} J_{i}$, then $f^{2}(x)=x$. From this description it follows that if each period of $f$ is a power of 2 , then $f$ is a period 2 homeomorphism. See the corollary.

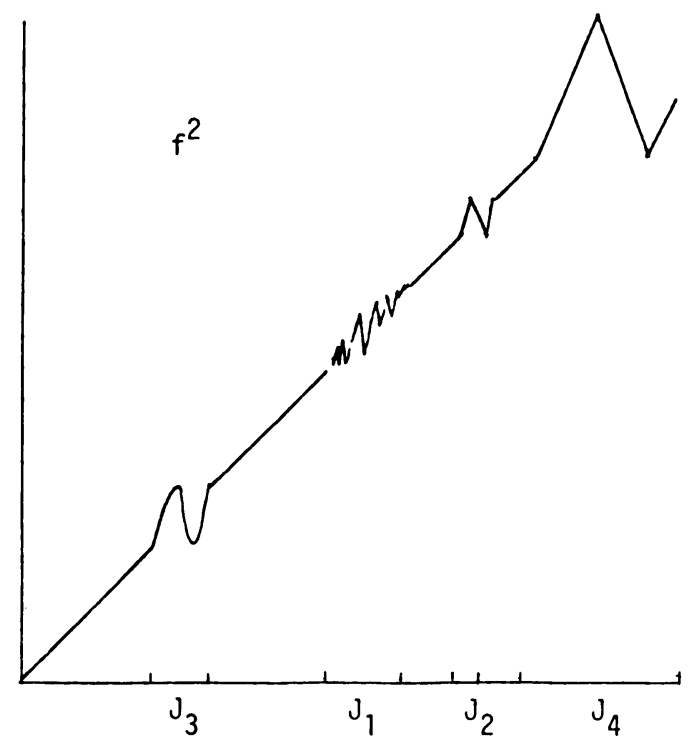

Received by the editors April 11, 1984 and, in revised form, October 31, 1984. 1980 Mathematics Subject Classification. Primary 58F13, 58F22, 55F20, 54H20. Key words and phrases. Periodic point, inverse limit, indecomposable continuum.

(C)1985 American Mathematical Society $0002-9939 / 85 \$ 1.00+\$ .25$ per page 
THEOREM. Suppose that $f: I \rightarrow I$ is continuous, and that the set of periodic points of $f$ is dense in I. Then there is a collection (perhaps finite or empty) $\left\{J_{1}, J_{2}, \ldots\right\}$ of mutually disjoint closed subintervals of I such that (i) $f^{2}\left(J_{i}\right)=J_{i}$, (ii) for each $i$, there is a point $x_{i} \in J_{i}$ such that $\left\{f^{4 n}\left(x_{i}\right) \mid n \geqslant 0\right\}$ is dense in $J_{i}$, and (iii) if $x \in I-\bigcup_{1}^{\infty} J_{i}$, then $f^{2}(x)=x$.

Definitions and terminology. Throughout, $I$ will denote a closed interval, and $f$ : $I \rightarrow I$ will be a continuous function. If $n$ is a nonnegative integer, $f^{n}$ is the $n$-fold composition of $f$. If $x \in I$, the orbit of $x, O(x)$ is $\left\{f^{n}(x) \mid n \geqslant 0\right\}$. The statement that $x$ is periodic means that there is an integer $n$ such that $f^{n}(x)=x$, and the statement that $x$ has period $n$ means that $n$ is a positive integer, $f^{n}(x)=x$, and if $k$ is an integer, $1 \leqslant k<n$, then $f^{k}(x) \neq x$.

Associated with $f: I \rightarrow I$ is the inverse limit space

$$
(I, f)=\left\{\left(x_{0}, x_{1}, \ldots\right) \mid f\left(x_{i+1}\right)=x_{i}\right\}
$$

with metric

$$
d\left(\left(x_{0}, x_{1}, \ldots\right),\left(y_{0}, y_{1}, \ldots\right)\right)=\sum_{i=0}^{\infty} \frac{\left|x_{i}-y_{i}\right|}{2^{i}} .
$$

In [B-M] and [B-M2] we began a study of the dynamics of functions on the interval by analyzing $(I, f)$. The theorem in this paper is a continuation of this program.

$(I, f)$ is a compact, connected metric space, and is an example of what Bing [Bi] has called a snakelike continuum. We will denote elements of $(I, f)$ by subbarred letters, as $\underline{x}=\left(x_{0}, x_{1}, \ldots\right)$. The projection maps $\Pi_{n}$, of $(I, f)$ onto $I$ given by $\Pi_{n}(\underline{x})=x_{n}$ are continuous. If $H$ is a subcontinuum (compact, connected subspace) of $(I, f)$ we will let $H_{n}$ denote $\Pi_{n}(H)$. Note that $H_{n}$ is a closed interval or point and that $f\left(H_{n+1}\right)=H_{n}$.

If $f: I \rightarrow I$, then $f$ induces a homeomorphism $\hat{f}:(I, f) \rightarrow(I, f)$ by $\hat{f}\left(\left(x_{0}, x_{1}, \ldots\right)\right)$ $=\left(f\left(x_{0}\right), x_{0}, x_{1}, \ldots\right)$. We will utilize the fact that the intersection of any collection of subcontinua of $(I, f)$ is a subcontinuum of $(I, f)$. See [Bi].

If $S$ is a continuum, the statement that $S$ is indecomposable means that $S$ is not the union of two of its proper subcontinua. If $S$ is indecomposable and $H$ is a subcontinuum of $S$, then $H$ contains no open set in $S$ [H-Y, pp. 139-141].

Finally, in Lemma 2, we will utilize the following important construction due to Bing [Bi]. Suppose that $(I, f)$ contains no indecomposable subcontinuum with interior. For each $\underline{x} \in(I, f)$ let $g_{\underline{x}}$ be the intersection of all subcontinua of $(I, f)$ that contain interiorly a subcontinuum that contains $\underline{x}$ in its interior. Then $g_{\underline{x}}$ is a subcontinuum of $(I, f)$. Furthermore, the sets $g_{\underline{x}}$ partition $(I, f)$, and if we let $G=\left\{g_{\underline{x}} \mid \underline{x} \in(I, f)\right\}$ with the quotient topology, then $G$ is an arc (i.e. homeomorphic with $I$ ). Moreover, $f$ induces a homeomorphism $\hat{f}$ of $G$ onto $G$ given by $\hat{f}\left(g_{\underline{x}}\right)=g_{\hat{f}(\underline{x})}$. Bing also shows that $g_{\underline{x}}$ does not have interior.

Lemma 1. Suppose that $g: I \rightarrow I$ is continuous, that $(I, g)$ is indecomposable, and that the periodic points of $g$ are dense in I. Then $g^{2}$ has a dense orbit. 
Proof. We will first argue that $g$ has a dense orbit. Suppose that $J$ is a subinterval of $I$ and that $x \in$ int $I$. We will show that there is an integer $m$ such that $x \in g^{m}(J)$. Let $p$ be a periodic point in int $J$. Suppose that $p$ has period $k$. Let $L=$ $\operatorname{cl}\left(\cup_{n=0}^{\infty} g^{n k}(J)\right)$. Suppose that $L \neq I$, and that there is a point $y \in I-L$ such that $g^{k}(y) \in L$. Then there is a periodic point $q \in I-L$ such that $g^{k}(q) \in L$. Suppose that the period of $q$ is $j$. Then $g^{j k}(q)=q$, but $g^{j k}(q) \in L$. This is a contradiction, and so $g^{-k}(L)=L$. Then we have $g^{k}(L)=L$, and $g^{-k}(L)=L$. Now, $(I, g)$ is homeomorphic with $\left(I, g^{k}\right)$, but since $g^{-k}(L)=L$, we have that $\left(L, g^{k}\right)$ is a proper subcontinuum of $\left(I, g^{k}\right)$ with interior. This contradicts the indecomposability of $(I, g)$ [H-Y, pp. 139-141]. Thus,

$$
L=\operatorname{cl}\left(\bigcup_{n=0}^{\infty} g^{n k}(J)\right)=I
$$

It follows that there is an integer $m$ such that $x \in g^{m}(J)$. This argument shows that if $x \in$ int $I$, then $\bigcup\left\{g^{-n}(x) \mid n \geqslant 0\right\}$ is dense in $I$. It follows that if $U$ is an open set in $I$, then $\bigcup_{n=0}^{\infty}\left\{g^{-n}(U) \mid n \geqslant 0\right\}$ is dense in $I$. Then using Lemma 3 of [A-Y] we have that $g$ has a dense orbit.

Now, since $g$ has a dense orbit, and $(I, g)$ is indecomposable, it follows from Theorem 3 of [B-M1] that $g^{2}$ has a dense orbit.

LEMMA 2. Suppose that the periodic points of $f$ are dense, and that $(I, f)$ contains no indecomposable subcontinua with interior. Then $f^{2}$ is the identity.

Proof. Since $(I, f)$ contains no indecomposable subcontinua with interior we may use Bing's construction [Bi]. That is, there is an upper semicontinuous decomposition space $G$ of $(I, f)$ into subcontinua and points such that (1) the decomposition space $G$ is an arc, and (2) no element of $G$ has interior. Furthermore, the homeomorphism $\hat{f}:(I, f) \rightarrow(I, f)$ induces a homeomorphism $\hat{\hat{f}}: G \rightarrow G$, by $\hat{f}\left(g_{\underline{x}}\right)$ $=g_{\hat{f}(\underline{x})}$.

Since the periodic points of $f$ are dense, we have that the periodic points of $\hat{f}$ are dense, and that the periodic points of $\hat{\hat{f}}$ are dense. Since $G$ is an arc and $\hat{\hat{f}}$ is a homeomorphism, it follows that $\hat{f}^{2}$ is the identity.

We will next argue that every element in the decomposition $G$ is a point. Suppose that $H$ is a subcontinuum of $(I, f)$ and $H \in G$. Then $f^{2}(H)=H$, and if $H_{0}=$ $\Pi_{0}(H)$, then $f^{2}\left(H_{0}\right)=f^{2}\left(\Pi_{0}(H)\right)=\Pi_{0}\left(\hat{f}^{2}(H)\right)=\Pi_{0}(H)=H_{0}$. Thus, $H_{0}$ is invariant under $f^{2}$. Furthermore, if $K \in G, K \neq H$, then $f^{2}\left(K_{0}\right)=K_{0}$, and so $H_{0} \cap K_{0}=\varnothing$. Then if $H \in G$ we have that $f^{-2}\left(H_{0}\right)=H_{0}$. Then $H=$ $\left\{\left(x_{0}, x_{1}, \ldots\right) \mid x_{2 n} \in H_{0}\right\}$. Now if int $H_{0} \neq \varnothing$, then $H$ has interior. This is a contradiction, and so $H_{0}$ is a point.

Now let $x \in I$, and let $\underline{x}$ be a point of $(I, f)$ with $\Pi_{0}(\underline{x})=x_{0}=x$. Let $g_{\underline{x}}$ be the element of $G$ containing $\underline{x}$. Then from the previous discussion we have that $g_{\underline{x}}=\{\underline{x}\}$ and that $\hat{f}^{2}\left(g_{\underline{x}}\right)=g_{\underline{x}}$. Thus, $\hat{f}^{2}(\underline{x})=\underline{x}$, and so $f^{2}(x)=x$. This establishes Lemma 2. 
Proof of Theorem. We suppose that the periodic points of $f$ are dense. Suppose that $H$ is an indecomposable subcontinuum of $(I, f)$ with interior. Since the periodic points of $f$ are dense, it follows that the periodic points of $\hat{f}$ are dense, and so there is a point $\underline{x} \in$ int $H$ which is periodic. Suppose that $\underline{x}$ has period $k$.

Consider $H \cap \hat{f}^{k}(H)$. This is a subcontinuum of $H$ with interior, and so $\hat{f}^{k}(H)=$ $H$. Then we have that $f^{k}\left(H_{0}\right)=f^{k}\left(\Pi_{0}(H)\right)=\Pi_{0}\left(\hat{f}^{k}(H)\right)=\Pi_{0}(H)=H_{0}$.

Consider the intervals $H_{0}, H_{1}, \ldots, H_{k-1}$. We have that $f\left(H_{i}\right)=H_{i-1}(\bmod k)$. Suppose that $i \neq j$ and that int $H_{i} \cap$ int $H_{j} \neq \varnothing$. We have then that $\hat{f}^{i}(H) \cap \hat{f}^{j}(H)$ is a subcontinuum of $\hat{f}^{i}(H)$ with interior. Since $\hat{f}^{i}(H)$ is indecomposable, we have that $\hat{f}^{i}(H)=\hat{f}^{j}(H)$ and so $H_{i}=H_{j}$. Thus the distinct intervals in $\left\{H_{0}, H_{1}, \ldots, H_{k-1}\right\}$ have disjoint interiors.

We will next show that there are at most two distinct intervals in the collection $\left\{H_{0}, H_{1}, \ldots, H_{k-1}\right\}$. Suppose the collection contains more than two distinct intervals. A consideration of cases reveals that there are integers $i, j, r$, and $s$ such that $H_{i}, H_{j}$, and $H_{r}$ are distinct, no interval in the collection lies between $H_{i}$ and $H_{j}$, and $H_{r}$ lies between $f^{s}\left(H_{i}\right)$ and $f^{s}\left(H_{j}\right)$. It then follows, since periodic points are dense and int $H_{r} \neq \varnothing$, that there is a periodic point $x$ between $H_{i}$ and $H_{j}$ such that $f^{s}(x) \in H_{r}$. That is, $x \notin \bigcup_{i=0}^{k-1} H_{i}, f^{s}(x) \in \bigcup_{i=0}^{k-1} H_{i}$, and $f^{p}(x)=x$ for some $p>0$. But this is impossible since $f\left(\bigcup_{i=0}^{k-1} H_{i}\right)=\bigcup_{i=0}^{k-1} H_{i}$. Thus, there are at most two distinct intervals in the collection $\left\{H_{0}, H_{1}, \ldots, H_{k-1}\right\}$, and we have $f^{2}\left(H_{i}\right)=H_{i}$ for all $i \geqslant 0$.

Now let $\left\{H^{1}, H^{2}, H^{3}, \ldots\right\}$ be the collection of all indecomposable subcontinua of $(I, f)$ with interior. For each $i$, let $J_{i}=\Pi_{0}\left(H^{i}\right)$. It follows, as before, that int $J_{i} \cap$ int $J_{m}=\varnothing$ if $i \neq m$.

From the previous argument we see that $f^{2}\left(J_{i}\right)=J_{i}$. Applying Lemma 1 to $f^{2} \mid J_{i}$ : $J_{i} \rightarrow J_{i}$, we see that there is a point $x_{i} \in J_{i}$ such that $\left\{f^{4 n}\left(x_{i}\right) \mid n \geqslant 0\right\}$ is dense in $J_{i}$.

Let $J=\operatorname{cl}\left(\bigcup_{i=1}^{\infty} J_{i}\right)$. Since $f^{2}\left(J_{i}\right)=J_{i}$ for all $i \geqslant 1$, it is clear that if $x \in J-\bigcup_{i=1}^{\infty} J_{i}$, then $f^{2}(x)=x$. Thus the theorem is established in the case that $J=I$.

Suppose $J \neq I$. Let $\left\{K_{\alpha}\right\}$ be the collection of all components of $J$. For each $\alpha, K_{\alpha}$ is a point or a closed interval. Now let $L$ be the decomposition space whose elements are the points in $L-J$ together with the $K_{\alpha}$. That is, $L=\{[x] \mid x \in I\}$ where $[x]=[y]$ if and only if $x=y$ or $x, y \in K_{\alpha}$ for some $\alpha$. It is clear that $L$, with the quotient topology, is an arc.

Let $P: I \rightarrow L$ be the projection map, $P(x)=[x]$. Then $P$ is a continuous and closed surjection. Furthermore, notice that $P$ is monotone, i.e. the inverse image of a continuum in $L$ is a continuum in $I$. Since $f$ respects the decomposition there is a uniquely defined continuous surjection $g: L \rightarrow L$ defined by $g([x])=[f(x)]$, such that $g \circ P=P \circ f$. Then $P$ induces a continuous surjection on inverse limit spaces $\bar{P}$ : $(I, f) \rightarrow(L, g)$ by $\bar{P}\left(\left(x_{0}, x_{1}, \ldots\right)\right)=\left(P\left(x_{0}\right), P\left(x_{1}\right), \ldots\right)$.

We will now show that $(L, g)$ contains no indecomposable subcontinua with interior. Suppose, to the contrary, that $N$ is an indecomposable subcontinuum of $(L, g)$ that has nonempty interior. Then, since $P$ is monotone, it follows that $\bar{P}$ is monotone, and hence $\bar{P}^{-1}(N)$ is a subcontinuum of $(I, f)$. It follows from Zorn's Lemma that there is a minimal subcontinuum $M$ of $(I, f)$ such that $P(M)=N$. 
If $M$ decomposes as $M=M^{1} \cup M^{2}, M^{1}$ and $M^{2}$ subcontinua of $M$, then $N=\bar{P}\left(M^{1}\right) \cup \bar{P}\left(M^{2}\right)$. Since $N$ is indecomposable, $\bar{P}\left(M^{1}\right)=N$ or $\bar{P}\left(M^{2}\right)=N$. But then, since $M$ is minimal, $M^{1}=M$ or $M^{2}=M$. Thus $M$ is indecomposable. We will show that $M$ has interior.

Let $C=\left\{\underline{x} \in(L, g) \mid x_{n} \neq P(J)\right.$ for all $\left.n \geqslant 0\right\}$. Then, since $P(J)$ is closed and has empty interior, $C$ is a closed subset of $(L, g)$ with empty interior. Thus, int $N \cap((L, g)-C)$ is a nonempty open subset of $(L, g)$. Each point in $L-P(J)$ has a unique preimage under $P$ and it follows that each point in $(L, g)-C$ has a unique preimage under $\bar{P}$. Thus $\bar{P}^{-1}$ (int $N \cap((L, g)-C)$ ) is an open subset of $M$.

We now have that $M$ is an indecomposable subcontinuum of $(I, f)$ with interior. Thus $M$ must be one of the $H^{i}$. But then $\bar{P}(M)=N$ is a single point and this contradicts the choice of $N$. Thus, $(L, g)$ has no indecomposable subcontinua with interior.

Since the periodic points under $f$ are dense in $I$, the periodic points under $g$ are dense in $L$. An application of Lemma 2 shows that $g^{2}$ is the identity on $L$. Thus $f^{2}$ is the identity on $I-\bigcup_{i=1}^{\infty} J_{i}$. This completes the proof of the theorem.

Corollary. Suppose that the periodic points of $f$ are dense, and that every period of $f$ is a power of 2. Then $f$ is a homeomorphism, and if $x \in I$, then $f^{2}(x)=x$. In particular, $f$ has no points of period other than 1 or 2 .

Proof. Suppose that there is a subinterval $J$ of $I$ such that $f^{2}(J)=J$, and a point $x \in J$ such that $\left\{f^{4 n}(x) \mid n \geqslant 0\right\}$ is dense in $J$. Then if $g=f^{2} \mid J$ we have that $g^{2}$ has a dense orbit on $J$. It follows from Theorem 13 of [B-M2] that $g$ has a point of odd period. Then, there is a point $x \in J$ which is periodic under $f$, and whose period under $f$ is not a power of 2 . This is a contradiction, and so the collection $\left\{J_{1}, J_{2}, \ldots\right\}$ in the conclusion of the theorem is empty. It follows then, that if $x \in I, f^{2}(x)=x$. This establishes the corollary.

\section{REFERENCES}

[A-Y] J. Auslander and J. Yorke, Interval maps, factors of maps, and chaos, Tôhoku Math. J. 32 (1980), $177-188$

[B-M1] M. Barge and J. Martin, Chaos, periodicity and snakelike continua, Trans. Amer. Math. Soc. 289 (1985), 355-365.

[B-M2] _ Dense orbits on the interval, Michigan Math. J. (submitted); Preprint available.

[Bi] R. H. Bing, Snakelike continua, Duke Math. J. 18 (1951), 653-663.

[H-Y] J. Hocking and G. Young, Topologv, Addison-Wesley, Reading, Mass., 1961.

Department of Mathematics, University of Wyoming, Laramie, Wyoming 82071 (Current address of Joe Martin)

Current address (Marcy Barge): Department of Mathematical Sciences, Montana State University, Bozeman, Montana 59715 